Molecules 2004, 9, 782-791

molecules

ISSN 1420-3049

http://www.mdpi.org

\title{
Restricting Magnetic Interaction Pathways in Polyoxometalate Salts of Cationic Nitronyl Nitroxide Free Radicals
}

\author{
Ana Tarazón, Carlos Giménez-Saiz, Carlos J. Gómez-García and Francisco M. Romero* \\ Institut de Ciència Molecular, Universitat de València, Dr. Moliner 50, 46100-Burjassot, Spain. Tel. \\ (+34) 963544859, Fax (+34) 963544859.
}

* Author to whom correspondence should be addressed; e-mail: fmrm@uv.es

Received: 1 June 2004 / Accepted: 17 June 2004 / Published: 31 August 2004

\begin{abstract}
Salts 1 and 2 that combine the $\left[\mathrm{W}_{6} \mathrm{O}_{19}\right]^{2-}$ Lindqvist anion with the cationic nitronyl nitroxide $(\mathrm{NN})$ free radicals $p$-MepyNN ${ }^{+}$and ${ }^{n} \mathrm{Bu}_{3} \mathrm{NCH}_{2} \mathrm{NN}^{+}$, respectively, have been synthesized and their structural and magnetic properties have been studied.
\end{abstract}

Keywords: Nitroxide radicals, polyoxometalates, magnetism.

\section{Introduction}

Ferromagnetism based on purely organic materials has raised a lot of interest during the last decade [1]. Most of the studies have been performed with nitronyl nitroxide ( $\mathrm{NN}$ ) radicals due to their persistence, stability and ease of functionalization [2]. Bulk ferromagnetism in these compounds arises from ferromagnetic interactions between open-shell molecules in the solid state. For a proper design of such materials, chemists need control over 1) the organization of molecules in the solid state and 2) the sign of magnetic interactions between neighbouring molecules. The first problem has been addressed by using the concepts of crystal engineering [3]. Thus, hydrogen bonding and other types of noncovalent interactions have been used to create specific crystal packings by design [4]. The second aspect is more delicate to control since the prediction of the type of interaction (ferro or antiferromagnetic) has not been solved to date. Usually, ferromagnetic interactions have been ascribed to intermolecular contacts between atoms carrying spin densities of opposite signs (McConnell I proposal) [5]. This model is an easy-to-use tool, since spin densities can be obtained experimentally (NMR or polarized neutron diffraction methods) in the solid state [6]. However, it has been shown that 
McConnell I model fails to predict the magnetic behaviour of numerous crystalline assemblies of NN radicals [7]. Theoretical investigations are now oriented to the calculation of the exchange interaction for pairs of radicals by $a b$ initio methods. An upper limit of $7 \AA$ for the intermolecular $\mathrm{O} \cdots \mathrm{O}$ distance inside a pair is generally considered. Cluster Heisenberg models comprising all the interactions within this limit are proposed and the corresponding Hamiltonian is then solved with the calculated exchange coupling parameters. Once the thermodynamic properties are obtained, they can be compared with the experimental values to check the validity of the model [8]. Although this approach can account for the magnetic properties of several NN-based solids, it fails to give the synthetic chemist some guidelines for the design of ferromagnetic compounds. It neglects also the fact that the interaction between two neighbouring molecules can proceed through a third one (for instance, in a bifurcated hydrogen bond) in the same way as the superexchange interaction that takes place through a bridging ligand in a binuclear metal complex.

The correlation between structure and magnetism in these compounds is hindered by the presence of many different magnetic interaction pathways in the solid. This problem can be addressed empirically, for instance, by comparison of isostructural radical-based solids showing different magnetic properties [9]. We propose here a different approach that consists in isolating the spin carriers through the synthesis of crystalline salts that combine bulky cationic free radicals with large anions, like polyoxometallates (POM). This strategy restricts the number of effective pathways and leads to simple exchange interaction patterns that can account for the magnetic behaviour of the sample. It affords also systems that can be useful in comparison with theoretical calculations.

\section{Results and Discussion}

The family of cationic NN free radicals of the $N$-alkylpyridinium type is well documented [10]. Their iodide salts can be easily prepared by $N$-alkylation of the corresponding pyridine NN derivative with methyl iodide. Salts with different complex anions are accessible through metathesis reactions. Also, the anionic counterpart can be an extended magnetically-ordered network [11]. In this context, we have reported on salts combining $p$-MepyNN cations (Scheme 1) and bimetallic oxalato-bridged lattices [12].

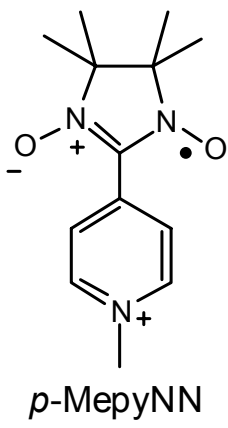




\section{Scheme 1.}



We have now developed a new synthetic strategy for the introduction of bulky cationic substituents in the $\alpha$-position of the NN moiety. It consists of the reaction between the NN free radical synthon $\mathrm{ClCH}_{2} \mathrm{NN}$ and a tertiary amine [13]. By choosing tributylamine as the nucleophilic agent, a sterically demanding tributylammonium-substituted free radical ${ }^{\mathrm{n}} \mathrm{Bu}_{3} \mathrm{NCH}_{2} \mathrm{NN}^{+}$cation can be obtained as the chloride salt (Scheme 1). Simple metathesis in the presence of POMs yields the hybrid POMcontaining NN salts. In this paper, we report on the synthesis, crystal structure and magnetic properties of the Lindqvist derivative $\left({ }^{\mathrm{n}} \mathrm{Bu}_{3} \mathrm{NCH}_{2} \mathrm{NN}\right)_{2}\left[\mathrm{~W}_{6} \mathrm{O}_{19}\right]$ and its comparison with the previously reported $(p-\text { MepyNN })_{2}\left[\mathrm{~W}_{6} \mathrm{O}_{19}\right]$ [14]. A detailed account of the crystal structure and magnetic properties of $(p-\mathrm{MepyNN})_{2}\left[\mathrm{~W}_{6} \mathrm{O}_{19}\right](\mathbf{1})$ has not been given in the literature and both aspects have been re-evaluated in the present work.

Figure 1. Projection of the crystal structure of 1 onto the $b c$ plane.




Compound 1 crystallizes in the centrosymmetric $P-1$ space group [15]. The Lindqvist anions lie on a center of symmetry and are surrounded by $p$-MepyNN ${ }^{+}$cations that form dimeric units by $\pi-\pi$ stacking of the pyridine fragments with an interplanar distance of ca. $3.6 \AA$ (Figure 1). The overall structure can also be viewed as alternating layers of $\left[\mathrm{W}_{6} \mathrm{O}_{19}\right]^{2-}$ anions and cationic free radicals. The organic layers are very well isolated, the shortest distance between nitroxide functionalities being greater than $8 \AA$.

The most relevant intermolecular contact within each layer $[\mathrm{O} 1 \cdots \mathrm{O} 2(1+x, y, z)=4.471(12) \AA]$ generates chains of radicals parallel to the $a$ axis. Two of these linear chains are linked together forming a ladder-like spin system (Figure 2). The steps of the ladder are defined by dimeric units of radicals packed around an inversion center, with short distances between nitroxide functions and methyl groups of adjacent imidazoline rings $[\mathrm{C} 10 \cdots \mathrm{O} 1(-x+1,-y+1,-z)=3.417(13) \AA]$. These contacts bring the nitroxide functionalities close $[\mathrm{O} 1 \cdots \mathrm{O} 1(-x+1,-y+1,-z)=4.963(16) \AA]$. The spin ladders are well separated by stacks of pyridinium cations and the shortest distance between nitroxide functions that belong to different ladders is $\mathrm{O} 2 \cdots \mathrm{O} 2(-x,-y+1,1-z)=5.865(16) \AA$. No other contacts between nitroxide groups below $7 \AA$ are found within the layers.

Figure 2. Projection of the crystal structure of 1 onto the $a c$ plane. The spin ladder system is shown by red rods. Dashed lines stand for interladder contacts.

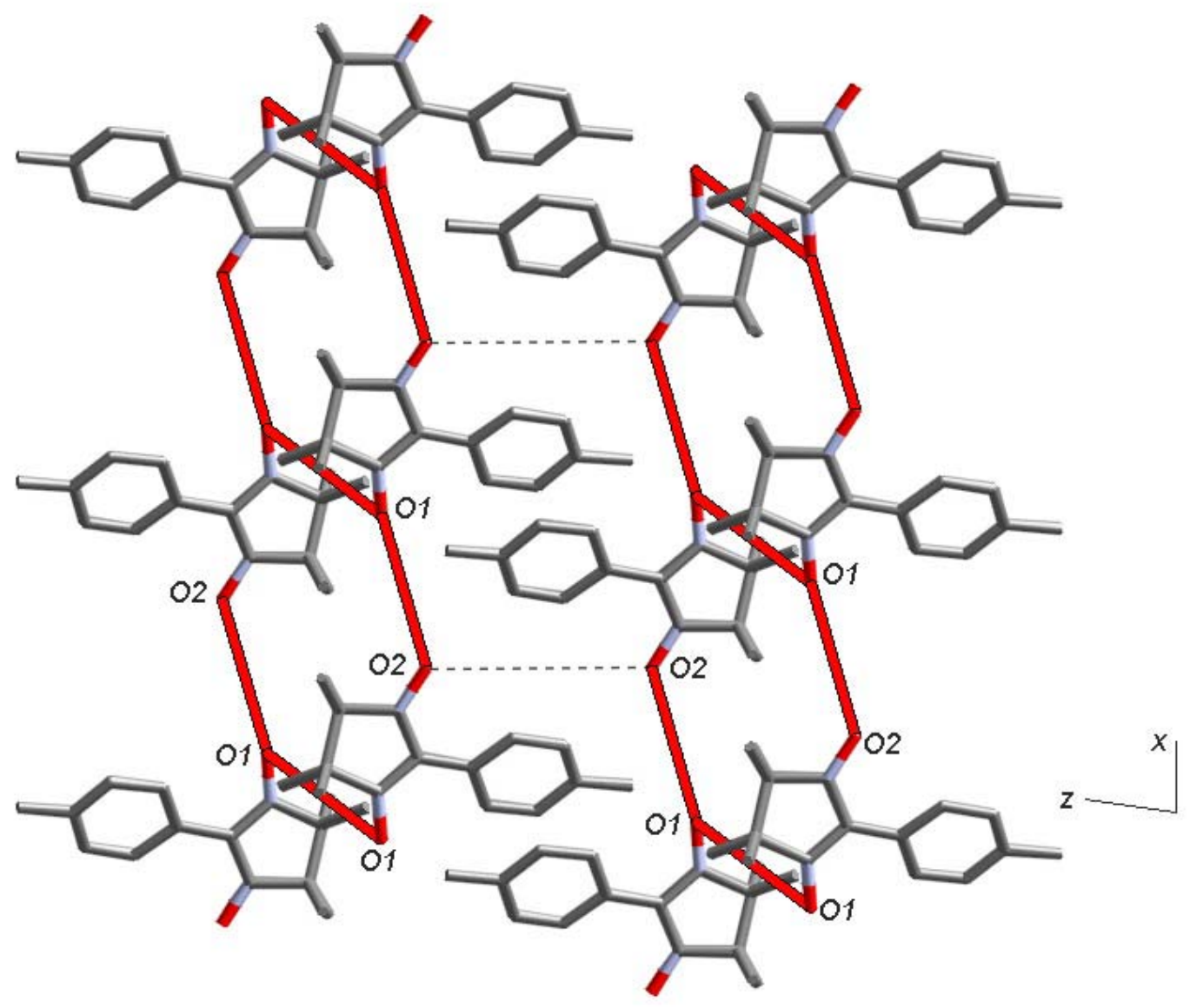


Magnetic susceptibility measurements of 1 have been recorded in the 2-300 K temperature range (Figure 3). At $300 \mathrm{~K}$, the product of molar magnetic susceptibility times temperature $(\chi T)$ equals 0.78 $\mathrm{emu} \cdot \mathrm{K} \cdot \mathrm{mol}^{-1}$, close to the expected value for two independent $\mathrm{S}=1 / 2$ spins per formula unit. Then, it shows a constant value on decreasing the temperature down to $50 \mathrm{~K}$. Further cooling leads to a small increase of $\chi T$ to a value of $0.84 \mathrm{emu} \cdot \mathrm{K} \cdot \mathrm{mol}^{-1}$ at $2.0 \mathrm{~K}$. This behaviour is characteristic of dominant ferromagnetic interactions. According to its structural packing, the magnetic properties of $\mathbf{1}$ can be analyzed by means of an $\mathrm{S}=1 / 2$ spin ladder model. Work along this direction is in progress. In the present work, we consider two approximations to this system: if the interaction along the steps of the ladder is neglected, the spin topology of the system can be described by the $S=1 / 2$ linear chain model [16]. The best-fit data using this approximation (solid line in Figure 3) yield an exchange coupling parameter $J=+0.20 \mathrm{~K}$. Alternatively, the interaction along the rungs of the ladder can be discarded, giving a simple dimeric unit. Least-squares fitting of the data (Bleaney-Bowers equation, dashed line) yields $J=+0.45 \mathrm{~K}$ [17]. The poor agreement between experimental and calculated data indicates that a spin ladder model with two types of interactions should be more reliable.

Figure 3. Temperature dependence of $\chi T$ for $\mathbf{1}$. Solid and dashed lines correspond to the best-fit data using linear chain and dimer models, respectively.



Compound 2 crystallizes in the centrosymmetric space group $P 2_{1} / c$ [18]. In this case, the crystal structure is not formed from segregated organic and inorganic layers and the Lindqvist anions are surrounded by ${ }^{\mathrm{n}} \mathrm{Bu}_{3} \mathrm{NCH}_{2} \mathrm{NN}$ cations in all three dimensions. Interestingly, only a few intermolecular

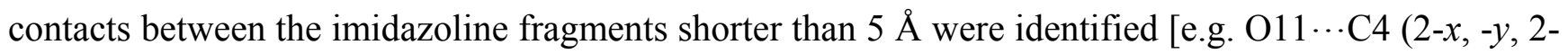
$z)=4.552(15) \AA]$. They involve a single centrosymmetric dimeric unit (Figure 4) that brings the nitroxide moieties to a distance of $011 \cdots \mathrm{O} 11(2-x,-y, 2-z)=6.068(16) \AA$. This is the shortest $\mathrm{O}_{\text {rad }} \cdots \mathrm{O}_{\text {rad }}$ distance found within this structure. Another relevant contact below the upper limit of $7 \AA$ 
is found between radicals related by the symmetry axis $(\mathrm{O} 11 \cdots \mathrm{O} 12(1-x, y-1 / 2,3 / 2-z)=6.713(10) \AA)$. These contacts organize zigzag chains that run parallel to the $b$ axis. The combination of the two interactions results in a hexagonal layered motif (Figure 5).

Figure 4. View of the centrosymmetric ${ }^{\mathrm{n}} \mathrm{Bu}_{3} \mathrm{NCH}_{2} \mathrm{NN}$ dimer.

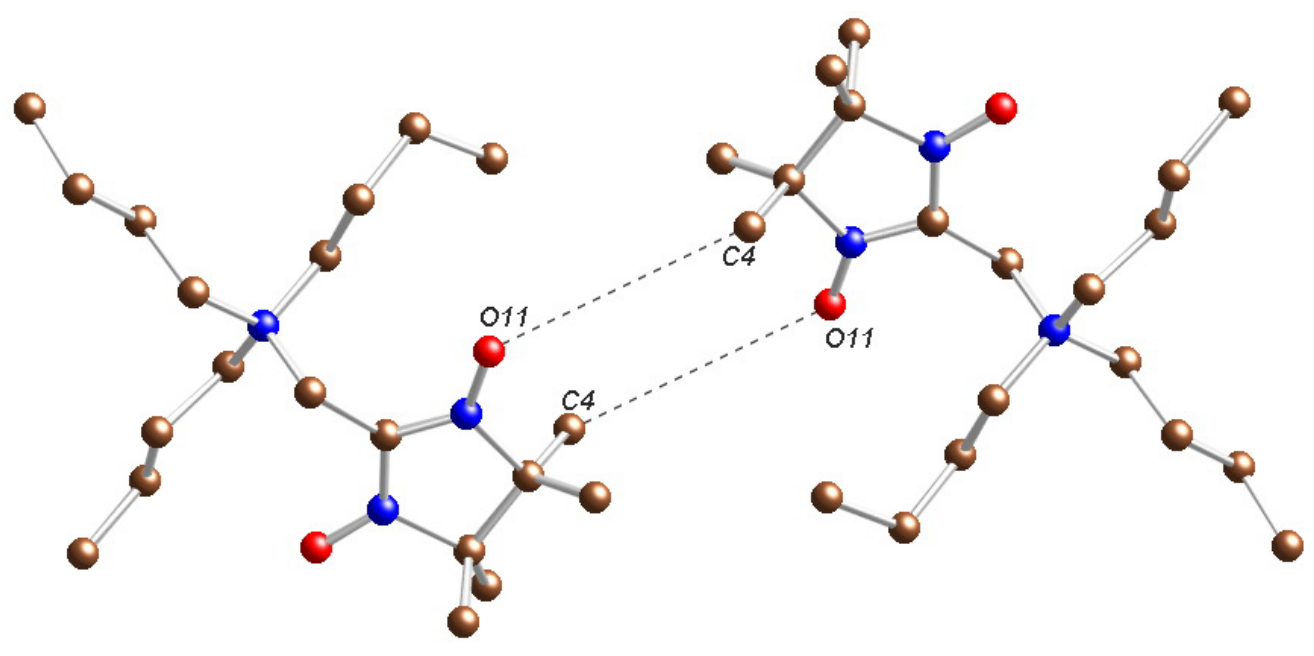

Figure 5. Projection of the crystal structure of 2 onto the $a b$ plane. Dimeric units are denoted by red rods. Dashed lines refer to zigzag chains along the $b$ axis.

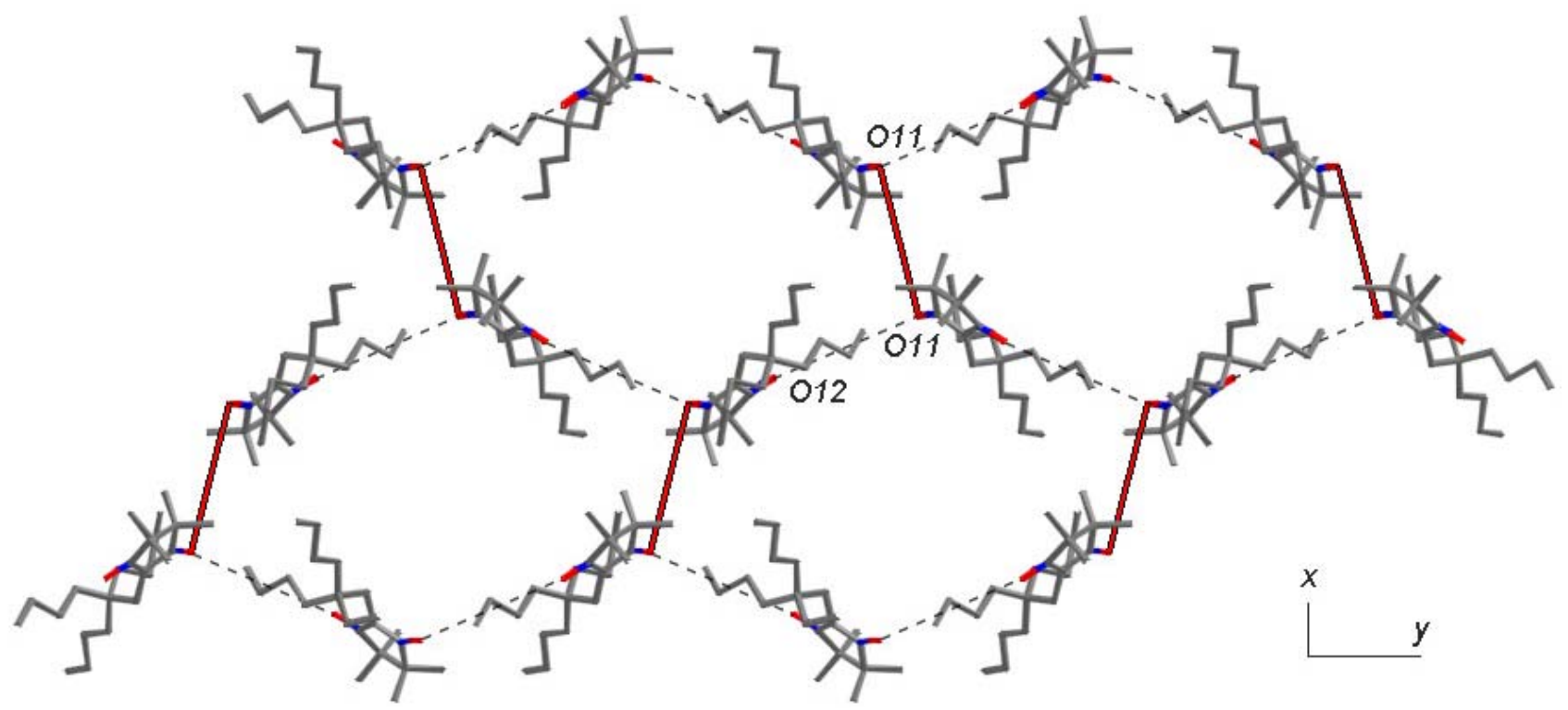

The $\chi T$ product of compound 2 (Figure 6a) equals $0.74 \mathrm{emu} \cdot \mathrm{K} \cdot \mathrm{mol}^{-1}$ at $300 \mathrm{~K}$, close to the calculated value for two uncorrelated $S=1 / 2$ spins per formula unit. It exhibits a constant value on decreasing the temperature down to $20 \mathrm{~K}$. Below this temperature a small increase of $\chi T$ to a maximum value of $0.78 \mathrm{emu} \cdot \mathrm{K} \cdot \mathrm{mol}^{-1}$ at $9.7 \mathrm{~K}$ is observed. Then, $\chi T$ decreases smoothly on further 
cooling and a value of $0.70 \mathrm{emu} \cdot \mathrm{K} \cdot \mathrm{mol}^{-1}$ is observed at $2.0 \mathrm{~K}$. This behaviour is characteristic of dominant ferromagnetic interactions to which a weak antiferromagnetic term is superimposed. According to its structural packing, the ferromagnetic interaction is expected to be correlated with the presence of the dimeric units, since this is the only close intermolecular contact in the solid. Obviously, a ferromagnetic dimer model fails to reproduce the entire $\chi T=\mathrm{f}(T)$ curve and the intradimer interaction has been estimated by least-squares fitting of the $\chi$ vs $T$ curve (Figure $6 \mathrm{~b}$ ) at temperatures above $5.5 \mathrm{~K}$. The best-fit parameter is $J=+0.25 \mathrm{~K}$.

Figure 6. Temperature dependences of $\chi T$ (a) and $\chi(\mathrm{b})$ for 2 . The solid line corresponds to the best-fit to data with the Bleaney-Bowers equation $(J=+0.25 \mathrm{~K})$.
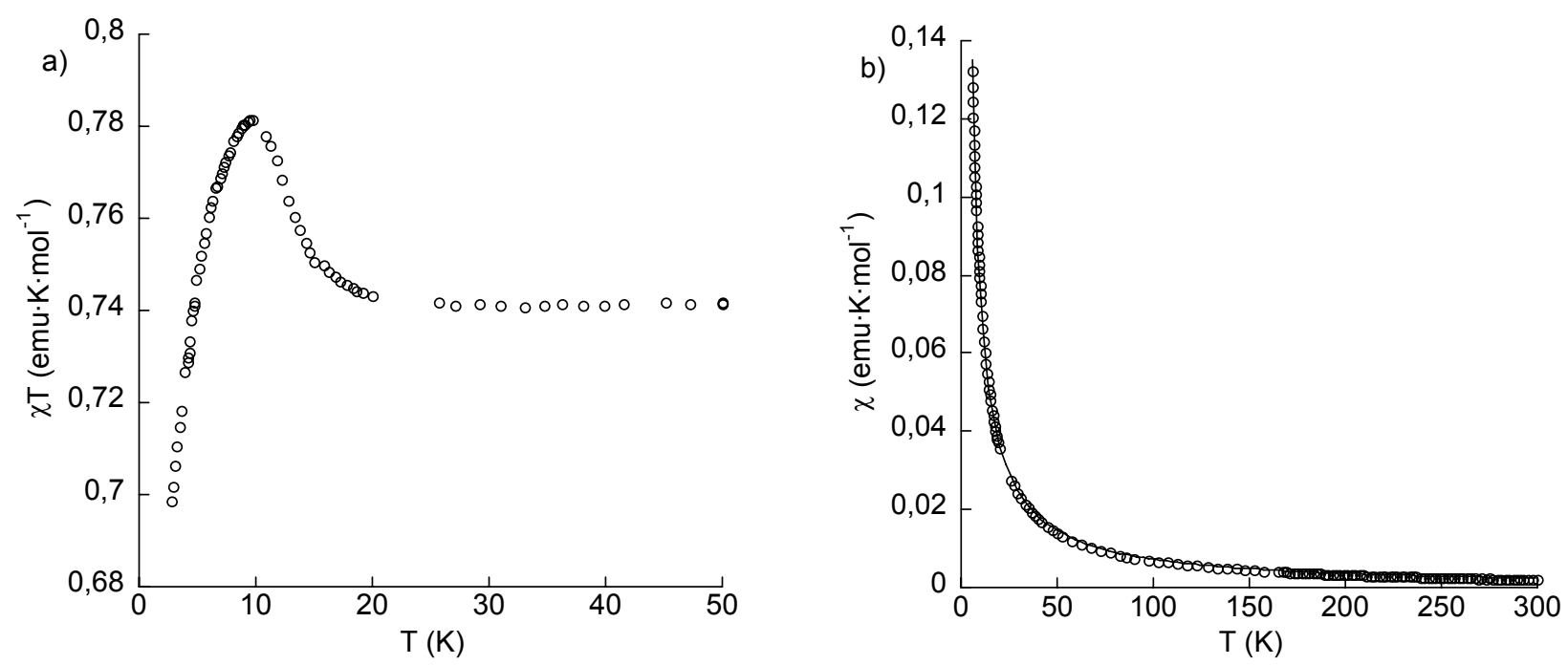

\section{Conclusions}

We have shown that it is possible to restrict the number of pathways for exchange coupling in free radical solids. This has been particularly the case for compound 2 , where the presence of bulky tributylammonium substituents and Lindqvist anions enables the formation of isolated dimeric entities. These compounds can be used as models for theoreticians interested in the calculation of magnetic interactions in systems of this kind. In the current examples dilution is also accompanied by a weakening of the intermolecular interactions. 


\section{Experimental}

\section{General}

IR transmission measurements of pressed $\mathrm{KBr}$ pellets were recorded in the $4000-400 \mathrm{~cm}^{-1}$ range at room temperature with a Nicolet Avatar 320 FT-IR spectrophotometer. ESI mass spectra were recorded on a Waters ZQ mass spectrometer using nitrogen as drying and nebulising gas. The equipment was calibrated with appropriate standard samples. CHN elemental analysis was carried out with a CE instruments EA 1110 CHNS analyzer. Variable temperature susceptibility measurements were carried out on polycrystalline samples in the 2-300 K temperature range at magnetic fields of 0.1 T (Quantum Design MPMS-XL-5 magnetometer equipped with a SQUID sensor). Diamagnetic contributions were corrected with Pascal constants. $p$-MepyNNClO $4,(\mathrm{TBA})_{2}\left[\mathrm{~W}_{6} \mathrm{O}_{19}\right](\mathrm{TBA}=$ tetrabutylammonium) and $\mathrm{ClCH}_{2} \mathrm{NN}$ were prepared by previously reported procedures $[13,19,20]$. All other chemicals were used as received unless otherwise stated. CCDC 241274 (compound 1) and CCDC 241275 (compound 2) contain the supplementary crystallographic data for this paper. These data can be obtained free of charge via www.ccdc.cam.ac.uk/conts/retrieving.html (or from the CCDC, 12 Union Road, Cambridge CB2 1EZ, UK; fax: +44 1223 336033; e-mail: deposit@ccdc.cam.ac.uk).

Preparation of (1-oxyl-3-oxide-4,4,5,5-tetramethylimidazolin-2-yl)methyltributylammonium chloride $\left({ }^{n} \mathrm{Bu}_{3} \mathrm{NCH}_{2} \mathrm{NNCl}\right)$

$\mathrm{ClCH}_{2} \mathrm{NN}(0.32 \mathrm{~g}, 1.6 \mathrm{mmol})$ was dissolved in anhydrous acetonitrile $(14 \mathrm{~mL})$. Tributylamine $(0.30$ $\mathrm{g}, 1.6 \mathrm{mmol}$ ) was added dropwise and the mixture was stirred under argon at $40^{\circ} \mathrm{C}$ for $24 \mathrm{~h}$. Then, the solvent was evaporated in vacuo and the residue was purified by chromatography on $\mathrm{SiO}_{2}$ using a 1$5 \%$ gradient of $\mathrm{MeOH}$ in $\mathrm{CH}_{2} \mathrm{Cl}_{2}$ as eluent to yield ${ }^{n} \mathrm{Bu}_{3} \mathrm{NCH}_{2} \mathrm{NNCl}(0.48 \mathrm{~g}, 78 \%)$ as a hygroscopic purple solid. IR (KBr pellets) $\mathrm{cm}^{-1}$ : 2962, 2930, 2874, 1458, $1376(\mathrm{~N}-\mathrm{O})$; ES-MS $\left(\mathrm{CH}_{3} \mathrm{CN}, 25 \mathrm{~V}\right)$ for $\mathrm{C}_{20} \mathrm{H}_{41} \mathrm{~N}_{3} \mathrm{O}_{2}\left(\mathrm{M}^{+}\right)$: Calcd 355.32; Found 355.05.

Preparation of 1-methyl-4-(1-oxyl-3-oxide-4,4,5,5-tetramethylimidazolin-2-yl)pyridinium nonadecaoxohexatungstate (1)

$p$ - $\mathrm{MepyNNClO}_{4}(0.10 \mathrm{~g}, 0.29 \mathrm{mmol})$ was dissolved in acetonitrile $(10 \mathrm{~mL})$. Solid $(\mathrm{TBA})_{2}\left[\mathrm{~W}_{6} \mathrm{O}_{19}\right]$ $(0.29 \mathrm{~g}, 0.14 \mathrm{mmol})$ was added at once and the mixture was stirred at room temperature for $30 \mathrm{~min}$ or until precipitation was completed. Single crystals were obtained by slow evaporation of the mother liquor. Yield: 85\%. IR (KBr pellets) $\mathrm{cm}^{-1}:$ 2964, 2936, 2876, 1469, 1372 (N-O), 975, 817; Elem. Anal. for $\left(\mathrm{C}_{13} \mathrm{H}_{19} \mathrm{~N}_{3} \mathrm{O}_{2}\right)_{2}\left[\mathrm{~W}_{6} \mathrm{O}_{19}\right]$ (Mr: 1905.65) Calcd: C, 16.39; H, 2.01; N, 4.41; Found: C, 16.50; H, 2.22; $\mathrm{N}, 4.35$. 
Preparation of (1-oxyl-3-oxide-4,4,5,5-tetramethylimidazolin-2-yl)methyltributylammonium nonadecaoxohexatungstate (2)

${ }^{\mathrm{n}} \mathrm{Bu}_{3} \mathrm{NCH}_{2} \mathrm{NNCl}(0.05 \mathrm{~g}, 0.13 \mathrm{mmol})$ was dissolved in acetonitrile $(6 \mathrm{~mL})$. Solid $(\mathrm{TBA})_{2}\left[\mathrm{~W}_{6} \mathrm{O}_{19}\right]$ $(0.12 \mathrm{~g}, 0.06 \mathrm{mmol})$ was added at once and the mixture was stirred at room temperature for $30 \mathrm{~min}$. Single crystals were obtained by slow evaporation of the resulting solution. Yield: $80 \%$. IR (KBr pellets) $\mathrm{cm}^{-1}$ : 2962, 2928, 2874, 1455, $1374(\mathrm{~N}-\mathrm{O})$, 975, 813; Elem. Anal. for $\left(\mathrm{C}_{20} \mathrm{H}_{41} \mathrm{~N}_{3} \mathrm{O}_{2}\right)_{2}\left[\mathrm{~W}_{6} \mathrm{O}_{19}\right]$ (Mr: 2218.15) Calcd: C, 22.68; H, 3.90; N, 3.97; Found: C, 22.96; H, 4.09; N, 3.92.

\section{References and Notes}

1. For an overview of purely organic magnetism see the Proceedings of the 8th International Conference on Molecule-based Magnets: Polyhedron 2003, 14-17, 1725.

2. Ullman, E. F.; Osiecki, J. H.; Boocock, D. G. B. J. Am. Chem. Soc. 1972, 94, 7049.

3. Desiraju, G. R. Angew. Chem. 1995, 107, 2541..

4. Cirujeda, J.; Mas, M.; Molins, E.; Lanfranc de Panthou, F.; Laugier, J.; Park, J. G.; Paulsen, C.; Rey, P.; Rovira, C.; Veciana, J. J. Chem. Soc., Chem. Commun. 1995, 709.

5. McConnell, H. M. J. Phys. Chem. 1963, 39, 1910.

6. Sporer, C.; Heise, H.; Wurst, K.; Ruiz-Molina, D.; Kopacka, H.; Jaitner, P.; Köhler, F.; Novoa, J. J.; Veciana, J. Chem. Eur. J. 2004, 10, 1355-1365; Zheludev, A.; Barone, V.; Bonnet, M.; Delley, B.; Grand, A.; Ressouche, E.; Rey, P.; Subra, R.; Schweizer, J. J. Am. Chem. Soc. 1994, 116, 2019.

7. Deumal, M.; Cirujeda, J.; Veciana, J.; Novoa, J. J. Chem. Eur. J. 1999, 5, 1631.

8. Deumal, M.; Bearpark, M. J.; Novoa, J. J.; Robb, M. A. J. Phys. Chem. A 2002, 106, 1299.

9. Romero, F. M.; Ziessel, R.; Bonnet, M.; Pontillon, Y.; Ressouche, E.; Schweizer, J. ; Delley, B.; Grand, A.; Paulsen, C. J. Am. Chem. Soc. 2000, 122, 1298.

10. (a) Awaga, K.; Inabe, T.; Nagashima, U.; Nakamura, T.; Matsumoto, M.; Kawabata, Y.; Maruyama, Y. Chem. Lett. 1991, 1777; (b) Yamaguchi, A.; Okuno, T.; Awaga, K. Bull. Chem. Soc. Jpn. 1996, 69, 875; (c) Michaut, C.; Ouahab, L.; Bergerat, P.; Kahn, O.; Bousseksou, A. J. Am. Chem. Soc. 1996, 118, 3610; (d) Imai, H.; Otsuka, T.; Naito, T.; Awaga, K.; Inabe, T. J. Am. Chem. Soc. 1999, 121, 8098.

11. (a) Stumpf, H. O.; Ouahab, L.; Pei, Y.; Grandjean, D.; Kahn, O. Science, 1993, 261, 447; (b) Stumpf, H. O.; Ou ahab, L.; Pei, Y.; Bergerat, P.; Kahn, O. J. Am. Chem. Soc. 1994, 116, 3866; (c) Vaz, M. G. F.; Pinheiro, L. M. M.; Stumpf, H. O.; Alcântara, A. F. C.; Golhen, S.; Ouahab, L.; Cador, O.; Mathonière, C.; Kahn, O. Chem. Eur. J. 1999, 5, 1486.

12. Ballester, G.; Coronado, E.; Giménez-Saiz, C.; Romero, F. M. Angew. Chem. Int. Ed. Engl. 2001, 40,792 . 
13. Similar alkylation reactions catalyzed by KI were previously reported. In our case, the reaction proceeds with good yields in the absence of catalyst: (a) Ulrich, G.; Turek, P.; Ziessel, R. Tetrahedron Lett. 1996, 37, 8755; (b) Ziessel, R.; Stroh, C. Org. Lett. 2003, 5, 2397.

14. (a) Rimbaud, C.; Ouahab, L.; Sutter, J. P.; Kahn, O. Mol. Cryst. Liq. Cryst. A 1997, 306, 67; (b) Ouahab, L. C.R. Acad. Sci. II C, 1998, 1, 369.

15. Crystal data for 1: $\mathrm{C}_{26} \mathrm{H}_{38} \mathrm{~N}_{6} \mathrm{O}_{23} \mathrm{~W}_{6}, \mathrm{M}=1905.72$, crystal dimensions: $0.40 \times 0.15 \times 0.15 \mathrm{~mm}^{3}$, triclinic, $P-1, a=8.263(5) \AA, b=11.427(5) \AA, c=12.065(16) \AA, \alpha=65.78(4)^{\circ}, \beta=79.40(7)^{\circ}, \gamma$ $=84.18(4)^{\circ}, V=1020.8(16) \AA^{3}, Z=1, \rho_{\text {calc }}=3.100 \mathrm{Mg} \mathrm{m}^{-3}, \mu(\mathrm{MoK} \alpha)=16.927 \mathrm{~mm}^{-1}$. A greenyellow dichroic prism-like single crystal of 1 was used for data collection on a Nonius Kappa CCD diffractometer $\left(3.74^{\circ}<2 \theta<59.96^{\circ}\right)$ equipped with a graphite monochromated MoK $\alpha$ radiation source $(\lambda=0.71073 \AA)$. Data collection was performed at 293(2) K. Of 6311 measured reflections, 5930 were independent $\left(R_{\text {int }}=0.0206\right)$ and used to refine 285 parameters. Final $R$ (I $>$ $2 \sigma(\mathrm{I})), R_{1}=0.0371, w R_{2}=0.1006$; final $R$ (all data), $R_{1}=0.0624, w R_{2}=0.1085$. Max. $/ \mathrm{min}$. residual peaks in the final difference map 3.440 and $-2.133 \mathrm{e} \cdot \AA^{-3}$.

16. Baker Jr., G. A.; Rushbrooke, G. S.; Gilbert, H. E. Phys. Rev. 1964, 135, A1272.

17. Bleaney, B; Bowers, K. D. Proc. Roy. Soc. (London) Ser. A 1952, 214, 451.

18. Crystal data for 2: $\mathrm{C}_{40} \mathrm{H}_{82} \mathrm{~N}_{6} \mathrm{O}_{23} \mathrm{~W}_{6}, \mathrm{M}=2118.22$, crystal dimensions: $0.15 \times 0.05 \times 0.05 \mathrm{~mm}^{3}$, monoclinic, $P 2_{1} / c, a=12.4870(4) \AA, b=18.1526(11) \AA, c=17.0350(9) \AA, \beta=131.587(5)^{\circ}, V=$ 2888.1(3) $\AA^{3}, Z=2, \rho_{\text {calc }}=2.436 \mathrm{Mg} \mathrm{m}^{-3}, \mu(\mathrm{MoK} \alpha)=11.979 \mathrm{~mm}^{-1}$. A purple prism-like single crystal of 2 was used for data collection on a Nonius Kappa CCD diffractometer $\left(4.48^{\circ}<2 \theta<\right.$ $\left.54.98^{\circ}\right)$ equipped with a graphite monochromated $\operatorname{MoK} \alpha$ radiation source $(\lambda=0.71073 \AA)$. Data collection was performed at 150(2) K. Of 54266 measured reflections, 6622 were independent $\left(R_{\text {int }}=0.1249\right)$ and used to refine 348 parameters. Final $R(\mathrm{I}>2 \sigma(\mathrm{I})), R_{1}=0.0502, w R_{2}=0.1195$; final $R$ (all data), $R_{1}=0.0673, w R_{2}=0.1298$. Max. $/$ min. residual peaks in the final difference map 3.837 and $-6.215 \mathrm{e} \cdot \AA^{-3}$.

19. Yamaguchi, A.; Awaga, K.; Inabe, T.; Nakamura, T.; Matsumoto, M.; Maruyama, Y. Chem. Lett. 1993, 1443.

20. Fournier, M. Inorg. Synth. 1990, 27, 80.

Sample Availability: Available from the authors.

C 2004 by MDPI (http://www.mdpi.org). Reproduction is permitted for noncommercial purposes. 